\title{
Gluon form factor decompositions from the worldline formalism
}

\author{
Naser Ahmadiniaz \\ Center for Relativistic Laser Science, Institute for Basic Science, Gwangju 61005, Korea \\ E-mail: Ahmadiniazeibs.re.kr

\section{Christian Schubert*} \\ Instituto de Física y Matemáticas, Universidad Michoacana de San Nicolás de Hidalgo \\ Apdo. Postal 2-82, C.P. 58040, Morelia, Michoacan, Mexico \\ E-mail: schuberteifm.umich.mx
}

\begin{abstract}
We summarize recent work on the application of the string-inspired worldline formalism to the derivation of gauge-invariant form factor decompositions of the QCD off-shell N-gluon amplitudes. This formalism allows one to achieve such decompositions by way of integration-by-parts, rather than the usual tedious analysis of the non-abelian off-shell Ward identities, and to combine the scalar, spinor and gluon loop cases. For the three-gluon case, we rederive the standard Ball-Chiu decomposition in an efficient way. For the four-gluon vertex, we obtain a new decomposition in terms of 19 tensors, of which only 14 have full four-point kinematics; the remaining five are the Ball-Chiu tensors reappearing as boundary terms. A particularly compact representation is obtained for the case of $\mathscr{N}=4$ SYM theory. We compare with the low-energy effective action.
\end{abstract}

Loops and Legs in Quantum Field Theory

24-29 April 2016

Leipzig, Germany

${ }^{*}$ Speaker. 


\section{The QCD N-gluon vertices}

Recent years have witnessed tremendous progress in the calculation of on-shell matrix elements in quantum field theory, as exemplified by many talks at this conference (see $[1,2]$ for recent reviews). For off-shell amplitudes, however, progress has been much slower. Off-shell information is necessary, e. g., for the full exploitation of the renormalization group, the study of the infrared properties of QCD [3], as well as for the matching of perturbative information with lattice data (see, e.g., [4]). Having explicit results for off-shell amplitudes, or at least well-organized integral representations for them, can also be highly useful for the construction of higher-loop amplitudes. Off-shell amplitudes generally depend on a large numbers of Lorentz invariants, so that, beyond the simplest cases, usually there is little hope for an explicit closed-form evaluation. The challenge is then rather to obtain integral representations that are suitable to numerical evaluation, and welladapted to the symmetries of the amplitude. In QCD, an important part of this task is to find a tensor decomposition in the polarization indices that harmonizes with the off-shell Ward identities.

Here, we will describe a formalism that allows one to obtain such "form factor decompositions" for the QCD $N$ - gluon amplitudes with an arbitrary number of gluons, starting from the "worldline path integral representation" of these amplitudes at the one-loop level. Thus in terms of standard field theory, we deal with the Feynman diagrams shown in Fig. 1 (here we show the spinor loop case).

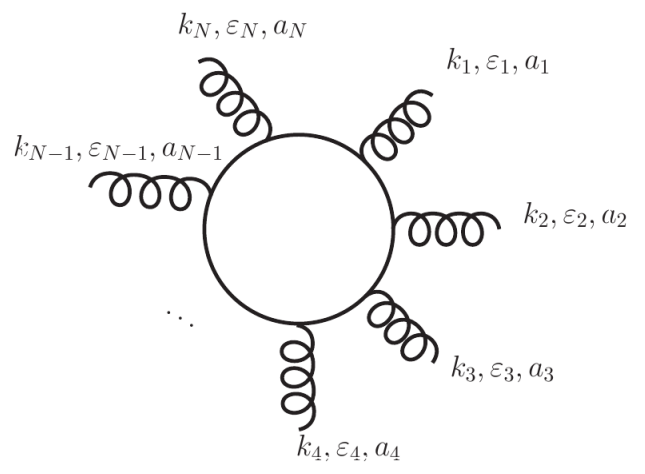

Figure 1: Diagram contributing to the N-gluon amplitudes.

We denote the one-loop off-shell one-particle irreducible ('1PI') N-gluon function or "vertex" by $\Gamma_{s \mu_{1} \ldots \mu_{N}}^{a_{1} a_{2} \cdots a_{N}}\left[k_{1}, \ldots, k_{N}\right]$, with $s=0, \frac{1}{2}, 1$ for the scalar, spinor, and gluon loop. Off-shell, the Ward identities for the gluon amplitudes are inhomogeneous, and map $N$ - point to $N-1$ - point:

$$
\begin{aligned}
k_{1}^{\mu_{1}} \Gamma_{s \mu_{1} \ldots \mu_{N}}^{a_{1} a_{2} \cdots a_{N}}\left[k_{1}, \ldots, k_{N}\right]= & -i g f_{a_{1} a_{2} c} \Gamma_{s \mu_{2} \ldots \mu_{N}}^{c a_{3} a_{4} \cdots a_{N}}\left[k_{1}+k_{2}, k_{3}, \cdots, k_{N}\right] \\
& -i g f_{a_{1} a_{3} c} \Gamma_{s \mu_{2} \ldots \mu_{N}}^{a_{2} c a_{4}}\left[\mu_{N}, k_{2}+k_{3}, \cdots, k_{N}\right]-\ldots \\
& \text { (+ possible ghost terms) }
\end{aligned}
$$

These identities hold for the scalar and spinor loop cases unambiguously and without ghost terms. For the gluon loop their precise form depends on the choice of the gauge-fixing: for a generic gauge-fixing ghost terms will appear on the right-hand side. However, the method presented here 
is based on a path integral representation of the gluon loop amplitudes using the background field method with quantum Feynman gauge [5, 6], which guarantees the absence of such terms [7] (alternatively, the same 'gauge-invariant vertices' can be obtained through the pinch-technique $[8,9])$.

\section{The Ball-Chiu decomposition of the three-gluon vertex}

For the three-gluon vertex, the following form factor decomposition was obtained by Ball and Chiu in $1980[10,11]$ through an explicit analysis of the Ward identity:

$$
\Gamma_{\mu_{1} \mu_{2} \mu_{3}}\left(k_{1}, k_{2}, k_{3}\right)=f^{a b c}\left\{A T_{A}+B T_{B}+C T_{C}+F T_{F}+H T_{H}+S T_{S}+\text { cyclic perm. }\right\},
$$

where

$$
\begin{aligned}
T_{A} & =g_{\mu_{1} \mu_{2}}\left(k_{1}-k_{2}\right) \mu_{3}, \\
T_{B} & =g_{\mu_{1} \mu_{2}}\left(k_{1}+k_{2}\right) \mu_{3}, \\
T_{C} & =-\left[\left(k_{1} k_{2}\right) g_{\mu_{1} \mu_{2}}-k_{1 \mu_{2}} k_{2 \mu_{1}}\right]\left(k_{1}-k_{2}\right)_{\mu_{3}}, \\
T_{F} & =\left[\left(k_{1} k_{2}\right) g_{\mu_{1} \mu_{2}}-k_{1 \mu_{2}} k_{2 \mu_{1}}\right]\left[k_{1 \mu_{3}}\left(k_{2} k_{3}\right)-k_{2 \mu_{3}}\left(k_{1} k_{3}\right)\right], \\
T_{H} & =-g_{\mu_{1} \mu_{2}}\left[k_{1 \mu_{3}}\left(k_{2} k_{3}\right)-k_{2 \mu_{3}}\left(k_{1} k_{3}\right)\right]+\frac{1}{3}\left(k_{1 \mu_{3}} k_{2 \mu_{1}} k_{3 \mu_{2}}-k_{1 \mu_{2}} k_{2 \mu_{3}} k_{3 \mu_{1}}\right), \\
T_{S} & =\frac{1}{3}\left(k_{1 \mu_{3}} k_{2 \mu_{1}} k_{3 \mu_{2}}+k_{1 \mu_{2}} k_{2 \mu_{3}} k_{3 \mu_{1}}\right) .
\end{aligned}
$$

It involves six universal tensor structures $T_{A}, T_{B}, T_{C}, T_{F}, T_{H}, T_{S}$ with scalar coefficient functions $A, B, C, F, H, S . \quad T_{A}$ is just the tree-level vertex, thus at tree-level one has $A=1$ with the other coefficient functions vanishing. $S$ turns out to vanish at one-loop [11] and presumably to all orders in perturbation theory [12]. Of the remaining ones, $F$ and $H$ possess true three-point kinematics, and are transversal, while $A, B, C$ have (pinched) two-point kinematics, and have a longitudinal part.

\section{The Bern-Kosower formalism}

A new perspective on the QCD gluon amplitudes is provided by string theory, where they appear as the infinite-string tension limit of certain string amplitudes. Along these lines, Bern and Kosower in the early nineties obtained the following "Bern-Kosower master formula" for the one-loop $N$ - gluon amplitudes [13, 14]:

$$
\begin{aligned}
& \Gamma^{a_{1} \ldots a_{N}}\left[k_{1}, \varepsilon_{1} ; \ldots ; k_{N}, \varepsilon_{N}\right]=(-i g)^{N} \operatorname{tr}\left(T^{a_{1}} \ldots T^{a_{N}}\right) \int_{0}^{\infty} d T(4 \pi T)^{-D / 2} e^{-m^{2} T} \\
& \quad \times\left.\int_{0}^{T} d \tau_{1} \int_{0}^{\tau_{1}} d \tau_{2} \ldots \int_{0}^{\tau_{N-2}} d \tau_{N-1} \exp \left\{\sum_{i, j=1}^{N}\left[\frac{1}{2} G_{B i j} k_{i} \cdot k_{j}-i \dot{G}_{B i j} \varepsilon_{i} \cdot k_{j}+\frac{1}{2} \ddot{G}_{B i j} \varepsilon_{i} \cdot \varepsilon_{j}\right]\right\}\right|_{\operatorname{lin}\left(\varepsilon_{1} \ldots \varepsilon_{\mathrm{N}}\right)} .
\end{aligned}
$$


As it stands, this is a parameter integral representation for the (color-ordered) $N$ - gluon vertex, with momenta $k_{i}$ and polarizations $\varepsilon_{i}$, induced by a scalar loop, in $D$ dimensions. Here $m$ and $T$ are the loop mass and proper-time, $\tau_{i}$ the location of the $i$ th gluon along the loop, and

$$
G_{B i j}=\left|\tau_{i}-\tau_{j}\right|-\frac{\left(\tau_{i}-\tau_{j}\right)^{2}}{T}, \dot{G}_{B}\left(\tau_{i}, \tau_{j}\right)=\operatorname{sign}\left(\tau_{i}-\tau_{j}\right)-2 \frac{\left(\tau_{i}-\tau_{j}\right)}{T}, \ddot{G}_{B}\left(\tau_{i}, \tau_{j}\right)=2 \delta\left(\tau_{i}-\tau_{j}\right)-\frac{2}{T} .
$$

In the Bern-Kosower formalism, this formula is a generating functional for the on-shell $N$ - gluon amplitudes for the scalar, spinor and gluon loop, via the application of the "Bern-Kosower rules":

1. For fixed $N$, expand the generating exponential, keeping only the terms that are linear in each polarization vector.

2. Use suitable integrations-by-parts ('IBP') to remove all second derivatives $\ddot{G}_{B i j}$.

3. Apply two types of pattern-matching rules :

- The "tree replacement rules", which generate the contributions of the missing reducible diagrams.

- The "loop replacement rules", which generate the integrands for the spinor and gluon loop from the one for the scalar loop.

\section{Strassler's worldline path integral approach}

Following the work of Bern and Kosower, Strassler [5] rederived the master formula and the loop replacement rules using worldline path integral representations of the gluonic effective actions $\Gamma[A]$. E.g. for the scalar loop $[15,5,16]$

$$
\Gamma[A]=\operatorname{tr} \int_{0}^{\infty} \frac{d T}{T} e^{-m^{2} T} \int \mathscr{D} x(\tau) \mathscr{P} e^{-\int_{0}^{T} d \tau\left(\frac{1}{4} \dot{x}^{2}+i g \dot{x} \cdot A(x(\tau))\right)},
$$

where $A_{\mu}=A_{\mu}^{a} T^{a}$ and $\mathscr{P}$ denotes path ordering. This rederivation made it also clear that the master formula and the loop replacement rules hold off-shell. However, reducible contributions have to be calculated separately in this approach.

In [17], Strassler then proceeded to a systematic study of the effect of the IBP procedure, and noted that it leads to the automatic appearance of gluon field strength tensors: namely, in the bulk it rearranges polarization vectors into the "abelian" part of the field strength tensor for gluon $i$,

$$
f_{i}^{\mu v} \equiv k_{i}^{\mu} \varepsilon_{i}^{v}-\varepsilon_{i}^{\mu} k_{i}^{v},
$$

and it also induces color commutators $\left[T^{a_{i}}, T^{a_{j}}\right]$ as boundary terms. Those fit together to produce full nonabelian field strength tensors

$$
F_{\mu \nu} \equiv F_{\mu \nu}^{a} T^{a}=\left(\partial_{\mu} A_{\nu}^{a}-\partial_{\nu} A_{\mu}^{a}\right) T^{a}+i g\left[A_{\mu}^{b} T^{b}, A_{\nu}^{c} T^{c}\right]
$$

in the low-energy effective action. Thus we see the emergence of gauge invariant tensor structures at the integrand level. This observation suggests that the worldline approach may be suitable for deriving well-organized form factor decompositions for gluonic amplitudes without the usual tedious analysis of the Ward identities, and we have found in a recent series of papers [12, 18, 19] that this is indeed the case. 


\section{Ball-Chiu from the master formula}

For starters, let us show how to rederive the Ball-Chiu decomposition (2.1), (2.2) from the Bern-Kosower master formula (3.1) [12]. For $N=3$, the master formula yields

$$
\begin{gathered}
\Gamma_{0}^{a_{1} a_{2} a_{3}}\left[k_{1}, \varepsilon_{1} ; k_{2}, \varepsilon_{2} ; k_{3}, \varepsilon_{3}\right]=(-i g)^{3} \operatorname{tr}\left(T^{a_{1}} T^{a_{2}} T^{a_{3}}\right) \int_{0}^{\infty} d T(4 \pi T)^{-D / 2} e^{-m^{2} T} \\
\times \int_{0}^{T} d \tau_{1} \int_{0}^{\tau_{1}} d \tau_{2}(-i)^{3} P_{3} e^{\left(G_{B 12} k_{1} \cdot k_{2}+G_{B 13} k_{1} \cdot k_{3}+G_{23} k_{2} \cdot k_{3}\right)}, \\
P_{3}=\dot{G}_{B 1 i} \varepsilon_{1} \cdot k_{i} \dot{G}_{B 2 j} \varepsilon_{2} \cdot k_{j} \dot{G}_{B 3 k} \varepsilon_{3} \cdot k_{k}-\ddot{G}_{B 12} \varepsilon_{1} \cdot \varepsilon_{2} \dot{G}_{B 3 k} \varepsilon_{3} \cdot k_{k}-\ddot{G}_{B 13} \varepsilon_{1} \cdot \varepsilon_{3} \dot{G}_{B 2 j} \varepsilon_{2} \cdot k_{j} \\
\quad-\ddot{G}_{B 23} \varepsilon_{2} \cdot \varepsilon_{3} \dot{G}_{B 1 i} \varepsilon_{1} \cdot k_{i}
\end{gathered}
$$

(repeated indices $i, j, k, \ldots$ are to be summed). To remove the term involving $\ddot{G}_{B 12} \dot{G}_{B 31}$, add

$$
-\frac{\partial}{\partial \tau_{2}}\left(\dot{G}_{B 12} \varepsilon_{1} \cdot \varepsilon_{2} \dot{G}_{B 31} \varepsilon_{3} \cdot k_{1} e^{\left(G_{B 12} k_{1} \cdot k_{2}+G_{B 13} k_{1} \cdot k_{3}+G_{23} k_{2} \cdot k_{3}\right)}\right) .
$$

In the abelian case this total derivative term would integrate to zero, but here due to the color ordering it produces (one half of) the term

$$
\operatorname{tr}\left(T^{a_{1}}\left[T^{a_{2}}, T^{a_{3}}\right]\right) \varepsilon_{3} \cdot f_{1} \cdot \varepsilon_{2} \dot{G}_{B 12} \dot{G}_{B 21} e^{G_{B 12} k_{1} \cdot\left(k_{2}+k_{3}\right)} .
$$

It involves only a two-point integral, with "pinched" momenta $k_{2}+k_{3}$. At this stage we have

$$
\begin{gathered}
\Gamma_{0}=\frac{g^{3}}{(4 \pi)^{\frac{D}{2}}} \operatorname{tr}\left(T^{a_{1}}\left[T^{a_{2}}, T^{a_{3}}\right]\right)\left(\Gamma_{0}^{\text {bulk }}+\Gamma_{0}^{\text {bound }}\right), \\
\Gamma_{0}^{\text {bulk }}=-\int_{0}^{\infty} \frac{d T}{T^{\frac{D}{2}}} e^{-m^{2} T} \int_{0}^{T} d \tau_{1} \int_{0}^{\tau_{1}} d \tau_{2}\left(Q_{3}^{3}+Q_{3}^{3}\right) \exp \left\{\sum_{i, j=1}^{3} \frac{1}{2} G_{B i j} k_{i} \cdot k_{j}\right\}, \\
\Gamma_{0}^{\text {bound }}=-\int_{0}^{\infty} \frac{d T}{T^{\frac{D}{2}}} e^{-m^{2} T} \int_{0}^{T} d \tau_{1} \dot{G}_{B 12} \dot{G}_{B 21}\left(\varepsilon_{3} \cdot f_{1} \cdot \varepsilon_{2} e^{G_{B 12} k_{1} \cdot\left(k_{2}+k_{3}\right)}+\text { cycl. }\right), \\
Q_{3}^{3}=\dot{G}_{B 12} \dot{G}_{B 23} \dot{G}_{B 31} \operatorname{tr}\left(f_{1} f_{2} f_{3}\right), \quad Q_{3}^{2}=\frac{1}{2} \dot{G}_{B 12} \dot{G}_{B 21} \operatorname{tr}\left(f_{1} f_{2}\right) \dot{G}_{B 3 i} \varepsilon_{3} \cdot k_{i}+2 \text { perm. }
\end{gathered}
$$

This is not yet Ball-Chiu: $Q_{3}^{3}$ corresponds to the form factor $H$, but $Q_{3}^{2}$ not to $F$; it is not even transversal. We can make $Q_{3}^{2}$ transversal by adding another total derivative term:

$$
-\frac{r_{3} \cdot \varepsilon_{3}}{r_{3} \cdot k_{3}} \frac{1}{2} \operatorname{tr}\left(f_{1} f_{2}\right) \frac{\partial}{\partial \tau_{3}}\left(\dot{G}_{B 12} \dot{G}_{B 21} e^{(\cdot)}\right)+2 \text { perm }
$$

Here $r_{i}$ is a reference momentum such that $r_{i} \cdot k_{i} \neq 0$. This transforms $Q_{3}^{2}$ into

$$
S_{3}^{2}:=\dot{G}_{B 12} \dot{G}_{B 21} \frac{1}{2} \operatorname{tr}\left(f_{1} f_{2}\right) \dot{G}_{B 3 k} \frac{r_{3} \cdot f_{3} \cdot k_{k}}{r_{3} \cdot k_{3}}+2 \text { perm. }
$$


which is transversal. With the cyclic choice of reference vectors $r_{1}=k_{2}-k_{3}$ etc. $S_{3}^{2}$ becomes the Ball-Chiu form factor $F$ indeed. The boundary terms match with the form factors $A, B, C$. So far this was all for the scalar loop, but the transition to the spinor and gluon loop cases can be done simply by the loop replacement rules. For the three-point case, those are [5, 16]:

$\underline{\text { Scalar } \rightarrow \text { Spinor: }}$

$$
\begin{aligned}
\dot{G}_{B i j} \dot{G}_{B j i} \rightarrow \dot{G}_{B i j} \dot{G}_{B j i}-G_{F i j} G_{F j i}, & \dot{G}_{B 12} \dot{G}_{B 23} \dot{G}_{B 31} \rightarrow \dot{G}_{B 12} \dot{G}_{B 23} \dot{G}_{B 31}-G_{F 12} G_{F 23} G_{F 31} . \\
\quad & \quad{ }_{\text {Scalar }} \rightarrow \text { Gluon: } \\
\dot{G}_{B i j} \dot{G}_{B j i} \rightarrow \dot{G}_{B i j} \dot{G}_{B j i}-4 G_{F i j} G_{F j i}, & \dot{G}_{B 12} \dot{G}_{B 23} \dot{G}_{B 31} \rightarrow \dot{G}_{B 12} \dot{G}_{B 23} \dot{G}_{B 31}-4 G_{F 12} G_{F 23} G_{F 31} .
\end{aligned}
$$

Here $G_{F i j}=\operatorname{sign}\left(\tau_{i}-\tau_{j}\right)$.

\section{Generalization to the $\mathrm{N}$-gluon case}

For the general $N$ - gluon case, the IBP procedure becomes highly ambiguous [20]. Nevertheless, a systematic investigation [18] showed that there exist two algorithms that are, in some sense, preferred: The first algorithm, leading to the " $Q$ - representation", uses only local total derivative terms, and makes the relation between the gluon amplitudes and the effective action particularly transparent; the second algorithm, leading to the ' $S$ - representation', uses both local and non-local total derivative terms and is "Ball-Chiu like" in that all bulk terms become manifestly transversal.

\section{The four-gluon vertex}

The application of this formalism to the four-gluon case has been completed only recently [19]. Although for an off-shell gauge boson amplitude one can a priori construct 138 tensors, it turns out that, up to permutations, the string-inspired formalism leads to a decomposition in terms of only 19 tensors. Moreover, of those only 14 have full four-point kinematics; the remaining five come from boundary terms, and those involve pinched momenta. In the $S$ - representation, they are precisely the Ball-Chiu form factors $A, B, C, F, H$ reappearing with pinched momenta as boundary terms. Here, we will list only the 14 "true" four-point tensors:

$$
\begin{aligned}
T_{P}^{4} & =\operatorname{tr}\left(f_{1} f_{2} f_{3} f_{4}\right), \quad T_{N P}^{4}=\operatorname{tr}\left(f_{1} f_{3} f_{2} f_{4}\right), \\
T_{P}^{22} & =\frac{1}{4} \operatorname{tr}\left(f_{1} f_{2}\right) \operatorname{tr}\left(f_{3} f_{4}\right), \quad T_{N P}^{22}=\frac{1}{4} \operatorname{tr}\left(f_{1} f_{3}\right) \operatorname{tr}\left(f_{2} f_{4}\right), \\
T_{P}^{3} & =\operatorname{tr}\left(f_{1} f_{2} f_{3}\right) \frac{r_{4} f_{4} k_{1}}{r_{4} k_{4}}, \quad T_{N P}^{3}=T_{P}^{3}\left(k_{1} \rightarrow k_{2}\right), \\
T_{\text {quart }}^{2 a d j} & =\frac{1}{2} \operatorname{tr}\left(f_{1} f_{2}\right) \frac{r_{3} f_{3} k_{1}}{r_{3} k_{3}} \frac{r_{4} f_{4} k_{1}}{r_{4} k_{4}}, \quad T_{\text {quart }}^{2 o p p}=\frac{1}{2} \operatorname{tr}\left(f_{1} f_{3}\right) \frac{r_{2} f_{2} k_{1}}{r_{2} k_{2}} \frac{r_{4} f_{4} k_{1}}{r_{4} k_{4}}, \\
T_{P}^{2 a d j} & =\frac{1}{2} \operatorname{tr}\left(f_{1} f_{2}\right) \frac{r_{3} f_{3} k_{2}}{r_{3} k_{3}} \frac{r_{4} f_{4} k_{1}}{r_{4} k_{4}}, \quad T_{N P}^{2 a d j}=\frac{1}{2} \operatorname{tr}\left(f_{1} f_{2}\right) \frac{r_{3} f_{3} k_{1}}{r_{3} k_{3}} \frac{r_{4} f_{4} k_{2}}{r_{4} k_{4}}, \\
T_{C}^{2 a d j} & =\frac{1}{2} \operatorname{tr}\left(f_{1} f_{2}\right) \frac{r_{3} f_{3} k_{4} r_{4} f_{4} k_{1}+\frac{1}{2} r_{3} f_{3} f_{4} r_{4} k_{4} k_{1}}{r_{3} k_{3} r_{4} k_{4}}, \quad T_{Z}^{2 a d j}=T_{C}^{2 a d j}\left(k_{1} \rightarrow k_{2}\right), \\
T_{P}^{2 o p p} & =\frac{1}{2} \operatorname{tr}\left(f_{1} f_{3}\right) \frac{r_{2} f_{2} k_{3}}{r_{2} k_{2}} \frac{r_{4} f_{4} k_{1}}{r_{4} k_{4}}, \quad T_{N P}^{2 o p p}=\frac{1}{2} \operatorname{tr}\left(f_{1} f_{3}\right) \frac{r_{2} f_{2} k_{4} r_{4} f_{4} k_{1}+\frac{1}{2} r_{2} f_{2} f_{4} r_{4} k_{4} k_{1}}{r_{2} k_{2} r_{4} k_{4}} .
\end{aligned}
$$




\section{Comparison with the effective action}

The low energy expansion of the one-loop QCD effective action induced by a loop particle of mass $m$ can be expanded as

$$
\Gamma[F]=\int_{0}^{\infty} \frac{d T}{T} \frac{\mathrm{e}^{-m^{2} T}}{(4 \pi T)^{D / 2}} \operatorname{tr} \int d x_{0} \sum_{n=2}^{\infty} \frac{(-T)^{n}}{n !} O_{n}[F],
$$

where $O_{n}(F)$ is a Lorentz and gauge invariant expression of mass dimension $2 n$. To lowest orders and for the scalar loop, one has [21, 22]

$$
\begin{aligned}
O_{2}= & -\frac{1}{6} g^{2} F_{\mu v} F_{\mu v} \\
O_{3}= & -\frac{2}{15} i g^{3} F_{\kappa \lambda} F_{\lambda \mu} F_{\mu \kappa}-\frac{1}{20} g^{2} D_{\lambda} F_{\mu v} D^{\lambda} F^{\mu v} \\
O_{4}= & +\frac{2}{35} g^{4} F_{\kappa \lambda} F_{\lambda \kappa} F_{\mu v} F_{v \mu}+\frac{4}{35} g^{4} F_{\kappa \lambda} F_{\lambda \mu} F_{\kappa v} F_{v \mu}-\frac{1}{21} g^{4} F_{\kappa \lambda} F_{\lambda \mu} F_{\mu v} F_{v \kappa}-\frac{8}{105} i g^{3} F_{\kappa \lambda} D_{\lambda} F_{\mu v} D_{\kappa} F_{v \mu} \\
& -\frac{6}{35} i g^{3} F_{\kappa \lambda} D_{\mu} F_{\lambda v} D^{\mu} F_{v \kappa}+\frac{11}{420} g^{4} F_{\kappa \lambda} F_{\mu v} F_{\lambda \kappa} F_{v \mu}+\frac{1}{70} g^{2} D_{\kappa} D_{\lambda} F_{\mu v} D^{\lambda} D^{\kappa} F_{v \mu}
\end{aligned}
$$

As a check, we have matched the low-energy limit of our results for the three and four-gluon amplitudes against this effective action, and found complete agreement [19].

\section{The case of $\mathscr{N}=4 \mathrm{SYM}$}

In the maximally supersymmetric $\mathscr{N}=4 \mathrm{SYM}$ theory the one-loop two - and three - gluon amplitudes vanish. The one-loop four-gluon vertex becomes extremely simple: all boundary terms cancel out, and the bulk term involves only the scalar box integral $B(1234)$ :

$$
\Gamma^{a_{1} a_{2} a_{3} a_{4}}=4 g^{4} \operatorname{tr}\left(T^{a_{1}} T^{a_{2}} T^{a_{3}} T^{a_{4}}\right) T_{8} B(1234)+\text { non }- \text { cyclic permutations } .
$$

Here $T_{8}$ is a tensor known to string theorists [23]

$$
\begin{aligned}
T_{8}= & \operatorname{tr}\left(f_{1} f_{2} f_{3} f_{4}\right)+\operatorname{tr}\left(f_{1} f_{2} f_{4} f_{3}\right)+\operatorname{tr}\left(f_{1} f_{3} f_{2} f_{4}\right) \\
& -\frac{1}{4} \operatorname{tr}\left(f_{1} f_{2}\right) \operatorname{tr}\left(f_{3} f_{4}\right)-\frac{1}{4} \operatorname{tr}\left(f_{1} f_{3}\right) \operatorname{tr}\left(f_{2} f_{4}\right)-\frac{1}{4} \operatorname{tr}\left(f_{1} f_{4}\right) \operatorname{tr}\left(f_{2} f_{3}\right) .
\end{aligned}
$$

\section{Summary and Outlook}

- We have shown how to use the string-inspired worldline formalism for generating form factor decompositions of the $N$ - gluon vertex well-adapted to the Ward identities, without actually using those identities. Although the derivation is at the one-loop level, these decompositions should be useful at higher loop orders, too, since the Ward identities are loop-independent.

- At the one-loop level, the formalism allows one to obtain also the coefficient functions of these form factors in terms of Schwinger parameter integrals directly from the Bern-Kosower master formula. Although this master formula is derived from the spin zero path integral, it contains also the information on the spinor and gluon loop cases. 
- We have carried out this program explicitly for the three- and four-point cases.

- In particular, we have obtained a four-point generalization of the Ball-Chiu decomposition in terms of 19 form factors, together with the corresponding one-loop parameter integrals.

- Presently the main limitation of the formalism is that, for the gluon loop case, it is still restricted to the background field gauge with quantum Feynman gauge.

- As a side remark, let us mention here that our four-point form factor decomposition in the abelian (four-photon) case reduces to only six instead of 19 tensors.

\section{References}

[1] H. Elvang and Y.-t. Huang, arXiv:1308.1697 [hep-th].

[2] L. J. Dixon, SLAC-PUB-15775, arXiv:1310.5353 [hep-ph].

[3] R. Alkofer, M. Q. Huber and K. Schwenzer, Eur. Phys. J. C 62 (2009) 761, arXiv:0812.4045 [hep-ph].

[4] M. Pelaez, M. Tissier and N. Wschebor, Phys.Rev. D 88 (2013) 125003, arXiv:1310.2594 [hep-th].

[5] M. J. Strassler, Nucl. Phys. B 385 (1992) 145, hep-ph/9205205.

[6] M. Reuter, M. G. Schmidt and C. Schubert, Ann. Phys. (N.Y.) 259 (1997) 313, hep-th/9610191.

[7] M. Binger and S. J. Brodsky, Phys. Rev. D 74 (2006) 054016, hep-ph/0602199.

[8] J. M. Cornwall and J. Papavassiliou, Phys. Rev. D 40 (1989) 3474.

[9] J. Papavassiliou, Phys. Rev. D 47 (1993) 4728.

[10] J. S. Ball and T. W. Chiu, Phys. Rev. D 22, 2542 (1980).

[11] J. S. Ball and T. W. Chiu, Phys. Rev. D 22 (1980) 2250, Erratum ibido 23 (1981) 3085.

[12] N. Ahmadiniaz and C. Schubert, Nucl. Phys. B 869 (2013) 417, arXiv:1210.2331 [hep-ph].

[13] Z. Bern and D. A. Kosower, Nucl. Phys. B 362 (1991) 389.

[14] Z. Bern and D. A. Kosower, Nucl. Phys. B 379 (1992) 451.

[15] R.P. Feynman, Phys. Rev. 80 (1950) 440.

[16] C. Schubert, Phys. Rept. 355 (2001) 73, arXiv:hep-th/0101036.

[17] M. J. Strassler, "Field theory without Feynman diagrams: a demonstration using actions induced by heavy particles", SLAC-PUB-5978 (1992) (unpublished).

[18] N. Ahmadiniaz, C. Schubert and V.M. Villanueva, JHEP 1301 (2013) 312, arXiv:1211.1821 [hep-th].

[19] N. Ahmadiniaz and C. Schubert, "String-inspired form factor decompositions of the four-gluon vertex", in preparation.

[20] C. Schubert, Eur. Phys. J. C 5 (1998) 693, hep-th/9710067.

[21] A. van de Ven, Nucl. Phys. B 250 (1985) 593.

[22] D. Fliegner, P. Haberl, M.G. Schmidt and C. Schubert, Ann. Phys. (N.Y.) 264 (1998) 51, hep-th/9707189.

[23] J. Broedel and L. Dixon, JHEP 1210 (2012) 091, arXiv:1208.0876 [hep-th]. 